Int. Journal of Math. Analysis, Vol. 8, 2014, no. 20, 973 - 993

HIKARI Ltd, www.m-hikari.com

http://dx.doi.org/10.12988/ijma.2014.44101

\title{
New Approach to Classify Second Order Linear Delay Differential Equations With Constant Coefficients to Solvable Lie Algebra
}

\author{
Laheeb Muhsen \\ Department of Mathematical Sciences \\ University Technology Malaysia \\ 81310 Skudai, Johor, Malaysia \\ Normah Maan ${ }^{1}$ \\ Department of Mathematical Sciences \\ University Technology Malaysia \\ 81310 Skudai, Johor, Malaysia
}

Copyright (c) 2014 Laheeb Muhsen and Normah Maan. This is an open access article distributed under the Creative Commons Attribution License, which permits unrestricted use, distribution, and reproduction in any medium, provided the original work is properly cited.

\begin{abstract}
The quest of developing efficient and accurate classification scheme for solving second order differential equations (DE) with various coefficients to solvable Lie algebra is never ending. We report a novel classification scheme for second order linear delay differential equations (DDEs) of retarded type with constant coefficients to solvable Lie algebra. The absence of an equivalent transformation related with the change in variables is considered to determine the invariant of DDEs in solvable Lie algebra. The infinitesimal generator of DDEs is used to determine the associated symmetry group. The equations are solved and the solvable Lie algebras spanned by these parameters are obtained by satisfying the inclusion property. This method is further extended to classify neutral delay DE and all classes of second order linear DDEs
\end{abstract}

\footnotetext{
${ }^{1}$ Corresponding author
} 
with constant coefficients to solvable Lie algebra. The excellent features of the results and successful implementation suggest that our new classifier may constitute a basis for categorizing second order linear DDEs with constant coefficients to solvable Lie Algebra.

Mathematics Subject Classification: 34K06 and 17B30

Keywords: Delay differential equation, group analysis, Lie algebra

\section{Introduction}

Undoubtedly, differential equations (DE) has paramount importance in mathematics and other branches of science and engineering. There are several forms of DE such as ordinary, partial, stochastic, integro and delay to cite a few [1]. A number of mathematical models in physics, engineering sciences, biomathematics etc. $[2,3,4,5,6,7]$ lead to delay differential equations (DDEs). Often, researchers face difficulty in modeling DDEs due to lack of accurate classification scheme. Recently, the DDEs generated great interests in the mathematical formulation for solving a varieties of real life problem including population dynamics, coexistence of competitive micro-organisms, sugar quantity in blood, models of lasers and metal cutting, infectious disease, control problems, secure communication, traffic control and economics(see $[8,9,10,11,12])$. Moreover, the neutral delay type equations contain the derivative of the unknown function. The emergence of new phenomena makes the theory of neutral delay differential equations is even more complicated than non-neutral one. Therefore, it is mandatory to generalize the classification procedure uniquely to neutral delay with constant coefficient.

Group analysis introduced by Sophus Lie (see [13]) is considered to be an accurate method for studying the properties of DE, and the notation of continuous groups is called Lie groups. In the past, group analysis is widely exploited $[14,15,16]$. Ibragimov further applied this concept for classification of ordinary DE in terms of their symmetry groups [17]. Tanthanuch treated on definition of an admitted Lie group for functional DE [18] which helped Prapart Pue-on to introduce group classification for specific cases of second order delay DE of the form

$$
x^{\prime \prime}=f\left(t, x, x_{\tau}, x^{\prime}, x_{\tau}^{\prime}\right),
$$

by changing the variables and found the invariance of the Lie algebra in the space of changed variables. To determine the invariants of second order DDEs, consideration of Lie algebra [1] is inevitable because they do not posses an equivalent transformation related to the change of dependent and independent variables. Boyko studied Lie symmetries of a system of second order linear 
ordinary DE with constant coefficients in real and complex field to compute the dimension of maximal Lie invariance [19]. Later, Moyo reported the properties of symmetry Lie group of a system of two linear second order ordinary DE with constant coefficients and changed variables for providing group classification. He achieved the general solution of this system [20]. Despite many dedicated efforts the validity, versatility and successful implementation of a generalized classification scheme remain debatable.

We developed a new approach based on group analysis for complete classification of second order DDEs to solvable Lie algebra of the form

$$
x^{\prime \prime}=f\left(t, x, x_{\tau}, x^{\prime}, x_{\tau}^{\prime}\right),
$$

where $x=x(t), x_{\tau}=x(t-\tau), x^{\prime}=x^{\prime}(t), x_{\tau}^{\prime}=x^{\prime}(t-\tau)$ and $x^{\prime \prime}=x^{\prime \prime}(t)$. Furthermore, the generalization of this classification is carried out to second order neutral delay DEs of the form

$$
x^{\prime \prime}=f\left(t, x, x_{\tau}, x^{\prime}, x_{\tau}^{\prime}, x_{\tau}^{\prime \prime}\right),
$$

where $x_{\tau}^{\prime \prime}=x^{\prime \prime}(t-\tau)$.

This paper is organized as follows. Section 2 renders the details of Lie algebra and delay ordinary DE. The classification of second order retarded DDEs and neutral DDEs to solvable Lie algebra and its generalization with main results are described in Section 3.

\section{Preliminary Notes}

A classification of second order linear delay differential equations with constant coefficients to solvable Lie algebra is proposed. It is customary to provide some striking features of Lie algebra and delay differential equations.

Definition 2.1 [21] A Lie algebra $L$ is an n-dimensional solvable algebra if there exists a sequence that yields,

$$
L_{1} \subset L_{2} \subset \ldots \subset L_{n}=L
$$

here $L_{k}$ is called $k$-dimensional Lie algebra and $L_{k-1}$ is an ideal of $L_{k}, k=$ $1,2, \ldots, n$ in which two-dimensional Lie algebra are solvable.

Definition 2.2 [14] Let

$Q_{i}=\zeta_{s} \frac{\partial}{\partial x_{s}}$ and $Q_{j}=\eta_{s} \frac{\partial}{\partial x_{s}}, i, j=1,2, \ldots, r$ and $s=1,2, \ldots, n$

be two infinitesimal generators. The commutator $\left[Q_{i}, Q_{j}\right]$ of $Q_{i}$ and $Q_{j}$ is the first order operator

$$
\left[Q_{i}, Q_{j}\right]=Q_{i} Q_{j}-Q_{j} Q_{i}=\sum_{s}^{n} \sum_{m}^{n}\left(\zeta_{m} \frac{\partial \eta_{s}}{\partial x_{m}}-\eta_{m} \frac{\partial \zeta_{s}}{\partial x_{m}}\right) \frac{\partial}{\partial x_{s}} .
$$


Example 2.3 [14] Let

$$
Q_{1}=y \frac{\partial}{\partial x}, \quad Q_{2}=x^{2} \frac{\partial}{\partial x}+x y \frac{\partial}{\partial y}
$$

then

$$
\begin{gathered}
{\left[Q_{1}, Q_{2}\right]=Q_{1} x^{2} \frac{\partial}{\partial x}+Q_{1} x y \frac{\partial}{\partial y}-Q_{2} y \frac{\partial}{\partial x}} \\
=x y \frac{\partial}{\partial x}+y^{2} \frac{\partial}{\partial y} .
\end{gathered}
$$

The commutator [.,.] satisfies the properties of Lie algebra.

Definition 2.4 [22] A finite set of infinitesimal generator $\left\{Q_{1}, Q_{2}, \ldots, Q_{r}\right\}$ is said to be a basis for the Lie algebra $L$ if $Q_{i} \in L$ and

1. $Q_{1}, Q_{2}, \ldots, Q_{r}$ from a basis of the vector space $L$,

2. $\left[Q_{i}, Q_{j}\right]=c_{i j k} Q_{k}$.

The coefficients $c_{i j k}$ are called the structure constant of the Lie algebra, $i, j, k=$ $1,2, \ldots, r$.

Theorem 2.5 (Second fundamental theorem of Lie) [14]

Any two infinitesimal generators of an r-parameter Lie group, satisfy commutation relation of the form $\left[Q_{i}, Q_{j}\right]=c_{i j k} Q_{k}$, where $i, j$ and $k=1,2, \ldots, r$.

The commutator and the Jacobi identity together are capable to form real ( or complex ) linear combinations of the $Q_{i}$, providing these infinitesimal generator the structure of the Lie algebra associated with the Lie group. Furthermore, the given infinitesimal generators $Q_{1}, Q_{2}, \ldots, Q_{r}$ form a basis for a Lie algebra.

Theorem 2.6 (Third fundamental theorem of Lie) [14]

The structure constants satisfy the relations

$$
\begin{gathered}
c_{i j k}=-c_{j i k}, \\
c_{i j k} c_{k l m}+c_{j l k} c_{k i m}+c_{l i k} c_{k j m}=0
\end{gathered}
$$

where $i, j, k, l$ and $m=1,2, \ldots, r$. In other word, the structure constants determine the Lie algebra, and hence the Lie group. 
Now we consider the second order delay differential equations of the form

$$
x^{\prime \prime}=f\left(t, x, x_{\tau}, x^{\prime}, x_{\tau}^{\prime}\right)
$$

The infinitesimal generator

$$
X=\xi(t, x) \frac{\partial}{\partial t}+\eta(t, x) \frac{\partial}{\partial x},
$$

is admitted by a second order DDE (1). The corresponding canonical LieBäcklund operator has the form

$$
X=\zeta\left(t, x, x^{\prime}\right) \frac{\partial}{\partial x}
$$

where $\zeta=\eta-x^{\prime} \xi$. In Equation (1) the periodic property of $\xi$ which has to be satisfied for any solution of the Cauchy problem [23], implies the independence of the function $\xi$ on $x$, i.e., $\xi_{x}=0$. The determining equation (1) takes the form

$$
\left.\tilde{X}\left(x^{\prime \prime}-f\left(t, x, x_{\tau}, x^{\prime}, x_{\tau}^{\prime}\right)\right)\right|_{(1)}=0
$$

where

$$
\tilde{X}=\xi \frac{\partial}{\partial t}+\eta^{x} \frac{\partial}{\partial x}+\eta^{x_{\tau}} \frac{\partial}{\partial x_{\tau}}+\eta^{x^{\prime}} \frac{\partial}{\partial x^{\prime}}+\eta^{x^{\prime \tau}} \frac{\partial}{\partial x_{\tau}^{\prime}}+\eta^{x^{\prime \prime}} \frac{\partial}{\partial x^{\prime \prime}}
$$

and

$$
\begin{gathered}
\eta^{x}(t, x)=\eta(t, x), \\
\eta^{x_{\tau}}(t, x)=\eta^{\tau}\left(t-\tau, x_{\tau}\right), \\
\eta^{x^{\prime}}\left(t, x, x^{\prime}\right)=\eta_{1}\left(t, x, x^{\prime}\right)=\eta_{t}(t, x)+\left[\eta_{x}(t, x)-\xi_{t}(t, x)\right] x^{\prime}-\xi_{x}(t, x)\left(x^{\prime}\right)^{2}, \\
\eta^{x_{\tau}^{\prime}}\left(t, x_{\tau}, x_{\tau}^{\prime}=\eta_{1}^{\tau}\left(\left(t, x_{\tau}, x_{\tau}^{\prime}\right)=\eta_{t}^{\tau}\left(t-\tau, x_{\tau}\right)+\left[\eta_{x}^{\tau}\left(t-\tau, x_{\tau}\right)-\xi_{t}^{\tau}\left(t-\tau, x_{\tau}\right)\right] x_{\tau}^{\prime}\right.\right. \\
-\xi_{x}^{\tau}\left(t-\tau, x_{\tau}\right)\left(x_{\tau}^{\prime}\right)^{2}, \\
\eta^{x^{\prime \prime}}\left(t, x, x^{\prime}, x^{\prime \prime}\right)=\eta_{2}\left(t, x, x^{\prime}, x^{\prime \prime}\right)=\eta_{t t}(t, x)+\left[2 \eta_{t x}(t, x)-\xi_{t t}(t, x)\right] x^{\prime}+\left[\eta_{x x}(t, x)\right. \\
\left.-2 \xi_{t x}(t, x)\right]\left(x^{\prime}\right)^{2}-\xi_{x x}(t, x)\left(x^{\prime}\right)^{3}+\left[\eta_{x}(t, x)-2 \xi_{t}(t, x)\right] x^{\prime \prime}-3 \xi_{x}(t, x) x^{\prime} x^{\prime \prime}, \quad(5)
\end{gathered}
$$

\section{Second order linear delay differential equa- tion classification to solvable Lie algebra}

Here we present the classification of second order linear non-homogenous delay differential equations to solvable Lie algebra. 
The general form of second order linear retarded delay differential equation is given by,

$$
x^{\prime \prime}(t)+a(t) x^{\prime}(t)+b(t) x^{\prime}(t-\tau)+c(t) x(t)+d(t) x(t-\tau)=0,
$$

where $a(t), b(t), c(t), d(t)$ are arbitrary coefficients. The general infinitesimal generator associated with Equation (6) is of the form

$$
X=\xi(t, x) \frac{\partial}{\partial t}+\eta(t, x) \frac{\partial}{\partial x}+\eta^{\tau}\left(t-\tau, x_{\tau}\right) \frac{\partial}{\partial x_{\tau}} .
$$

From Equation (4) the second order extension

$$
X^{(2)}=X+\eta_{1}\left(t, x, x^{\prime}\right) \frac{\partial}{\partial x^{\prime}}+\eta_{2}\left(t, x, x^{\prime}, x^{\prime \prime}\right) \frac{\partial}{\partial x^{\prime \prime}}+\eta_{1}^{\tau}\left(t-\tau, x_{\tau}, x_{\tau}^{\prime}\right) \frac{\partial}{\partial x_{\tau}^{\prime}} .
$$

By combining (8) and (6), the following invariance conditions are achieved,

$$
\eta_{2}+a \eta_{1}+b \eta_{1}^{\tau}+c \eta+d \eta^{\tau}=0,
$$

where $\eta_{1}, \eta_{1}^{\tau}$ are the first extensions of ordinary and delay equations respectively, and the second extension of ODE are $\eta_{2}$.

Substituting (5), we get

$$
\begin{gathered}
\eta_{t t}+\left[2 \eta_{t x}-\xi_{t t}\right] x^{\prime}+\left[\eta_{x x}-2 \xi_{t x}\right]\left(x^{\prime}\right)^{2}-\xi_{x x}\left(x^{\prime}\right)^{3}+\left[\eta_{x}-2 \xi_{t}\right] x^{\prime \prime}-3 \xi_{x} x^{\prime} x^{\prime \prime}+a\left[\eta_{t}+\left[\eta_{x}-\xi_{t}\right] x^{\prime}\right. \\
\left.-\xi_{x}\left(x^{\prime}\right)^{2}\right]+b\left[\eta_{t}^{\tau}+\left[\eta_{x}^{\tau}-\xi_{t}^{\tau}\right] x_{\tau}^{\prime}-\xi_{x}^{\tau}\left(x_{\tau}^{\prime}\right)^{2}\right]+c \eta+d \eta^{\tau}=0 .
\end{gathered}
$$

Equating the first and second order coefficients of various monomials of $x$ and $x_{\tau}$, the following determining equations for the symmetry group of Equation (6) are acquired. (6).

Table 1: The determining equations for the symmetry group of Equation

\begin{tabular}{|c|l|c|}
\hline monomial & coefficient & number of equation \\
\hline 1 & $\eta_{t t}+a \eta_{t}+b \eta_{t}^{\tau}+c \eta+d \eta^{\tau}=0$ & $a_{1}$ \\
\hline$x^{\prime}$ & $2 \eta_{t x}-\xi_{t t}+a\left[\eta_{x}-\xi_{t}\right]=0$ & $a_{2}$ \\
\hline$\left(x^{\prime}\right)^{2}$ & $\eta_{x x}-2 \xi_{t x}-a \xi_{x}=0$ & $a_{3}$ \\
\hline$\left(x^{\prime}\right)^{3}$ & $\xi_{x x}=0$ & $a_{4}$ \\
\hline$x^{\prime} x^{\prime \prime}$ & $\xi_{x}=0$ & $a_{5}$ \\
\hline
\end{tabular}




\begin{tabular}{|c|l|c|}
\hline monomial & coefficient & number of equation \\
\hline$x^{\prime \prime}$ & $\eta_{x}-2 \xi_{t}=0$ & $a_{6}$ \\
\hline$x_{\tau}^{\prime}$ & $b\left[\eta_{x}^{\tau}-\xi_{t}^{\tau}\right]=0$ & $a_{7}$ \\
\hline$\left(x_{\tau}^{\prime}\right)^{2}$ & $b \xi_{x}^{\tau}=0$ & $a_{8}$ \\
\hline
\end{tabular}

From $\left(a_{5}\right)$ and onward $\xi$ does not depend on $x$. In $\left(a_{3}\right), \eta$ is linear in $x$, meaning $\eta=g(t) x+h(t)$ with $g(t)$ and $h(t)$ are arbitrary functions. In $\left(a_{6}\right), \xi_{t}=\frac{1}{2} g . \quad$ In $\left(a_{8}\right)$, since $b \neq 0$ and $\xi_{x}^{\tau}=0$ implies that $\xi^{\tau}$ does not depend on $x$. The periodic property achieves $\xi=\xi^{\tau}$ and $\xi_{t}=\xi_{t}^{\tau}$. In $\left(a_{7}\right)$, $\eta^{\tau}=\frac{1}{2} g(t) x+k(t-\tau)$ with $k(t-\tau)$ as arbitrary function.

Case 1 Here $a, b, c$, and $d$ are arbitrary constant not equal zero.

From $\left(a_{1}\right), g_{t t} x+h_{t t}+a g_{t} x+a h_{t}+\frac{b}{2} g_{t} x+b k_{t}+c g x+c h+\frac{d}{2} g x+d k=0$. Equating the coefficients of the various monomials one obtains,

$$
\begin{aligned}
g_{t t}+\left(a+\frac{b}{2}\right) g_{t}+\left(c+\frac{d}{2}\right) g & =0 . \\
h_{t t}+a h_{t}+b k_{t}+c h+d k & =0 .
\end{aligned}
$$

Which means that $g(t), h(t)$ and $k(t-\tau)$ are the solutions of (6). From (9),

$$
g=-\frac{g_{t t}+\left(a+\frac{b}{2}\right) g_{t}}{c+\frac{d}{2}}
$$

but $g=2 \xi_{t}$,

$$
\xi_{t}=-\frac{\xi_{t t t}+\left(a+\frac{b}{2}\right) \xi_{t t}}{c+\frac{d}{2}},
$$

this implies that

$$
\xi=-\frac{\xi_{t t}+\left(a+\frac{b}{2}\right) \xi_{t}}{c+\frac{d}{2}}+c_{1} .
$$

Let $a+\frac{b}{2}=B$, and $c+\frac{d}{2}=D$, then,

$$
\xi=c_{1}-\frac{\frac{1}{2} g_{t}+\frac{1}{2} B g}{D} .
$$

Now,

$$
g=c_{2}-\frac{1}{B} g_{t}-\frac{2 D}{B} \xi .
$$


This yield $\eta=\left(c_{2}-\frac{1}{B} g_{t}-\frac{2 D}{B} \xi\right) x+h$ and $\eta^{\tau}=\left(c_{3}-\frac{1}{2 B} g_{t}-\frac{D}{B} \xi\right) x+k$, where $c_{i}, i=1,2,3$ are arbitrary constants.

Recall Equation (7), the general infinitesimal generator for (6) gives,

$$
X=\xi \frac{\partial}{\partial t}+\eta \frac{\partial}{\partial x}+\eta^{\tau} \frac{\partial}{\partial x_{\tau}}
$$

Let $x_{\tau}=u$, then

$X=\left[c_{1}-\frac{g_{t}+B g}{2 D}\right] \frac{\partial}{\partial t}+\left[\left(c_{2}-\frac{1}{B} g_{t}-\frac{2 D}{B} \xi\right) x+h\right] \frac{\partial}{\partial x}+\left[\left(c_{3}-\frac{1}{2 B} g_{t}-\frac{D}{B} \xi\right) x+k\right] \frac{\partial}{\partial u}$.

The Lie algebra of (6) is spanned by the following three infinitesimal generater corresponding to each parameters $c_{i}, i=1,2,3$.

$$
\begin{gathered}
Q_{1}=\frac{\partial}{\partial t}, \quad Q_{2}=x \frac{\partial}{\partial x}, \quad Q_{3}=x \frac{\partial}{\partial u} \\
Q_{4}=-\left(\frac{1}{2 D} g_{t}+\frac{B}{2 D} g\right) \frac{\partial}{\partial t}+\left[h(t)-x\left(\frac{1}{B} g_{t}+\frac{2 D}{B} \xi\right)\right] \frac{\partial}{\partial x}+\left[k(t)-x\left(\frac{1}{2 B} g_{t}+\frac{D}{B} \xi\right)\right] \frac{\partial}{\partial u},
\end{gathered}
$$

Where $Q_{4}$ is an infinite dimensional Lie subalgebra. The commutator table is given by,

\begin{tabular}{|c|l|c|l|}
\hline & $Q_{1}$ & $Q_{2}$ & $Q_{3}$ \\
\hline$Q_{1}$ & 0 & 0 & 0 \\
\hline$Q_{2}$ & 0 & 0 & $Q_{3}$ \\
\hline$Q_{3}$ & 0 & $-Q_{3}$ & 0 \\
\hline
\end{tabular}

Here $L_{3}=\left\{Q_{1}, Q_{2}, Q_{3}\right\}$ is spanned by $Q_{1}, Q_{2}, Q_{3}$ is the Lie algebra of (6). The subspace $L_{1}=\left\{Q_{1}\right\}, L_{2}=\left\{Q_{1}, Q_{2}\right\}$ are one, two dimensional Lie subalgebra of $L_{3}$ respectively. Furthermore, they obey the inclusion property: $L_{1} \subset L_{2} \subset L_{3}$, and hence $L_{3}$ is a solvable Lie algebra of (6).

Remark 3.1 If $c=0$ or $d=0$, the same result is obtained.

Case 2 If $b=1$, and $a=c=d=0$. The Equation (6) classified to Lie algebra takes the form,

$$
Q_{1}=t \frac{\partial}{\partial t}+t x\left(\frac{\partial}{\partial x}+\frac{1}{2} \frac{\partial}{\partial u}\right), \quad Q_{2}=\frac{\partial}{\partial t}+x\left(\frac{\partial}{\partial x}+\frac{1}{2} \frac{\partial}{\partial u}\right), \quad Q_{3}=\frac{\partial}{\partial u},
$$




$$
Q_{4}=-g \frac{\partial}{\partial t}+(h-x \xi) \frac{\partial}{\partial x}-\left(h_{t}+\frac{1}{2} x \xi\right) \frac{\partial}{\partial u} .
$$

Now, $Q_{4}$ is an infinite dimensional Lie subalgebra and the corresponding commutator table yields,

\begin{tabular}{|c|l|c|l|}
\hline & $Q_{1}$ & $Q_{2}$ & $Q_{3}$ \\
\hline$Q_{1}$ & 0 & $Q_{2}$ & 0 \\
\hline$Q_{2}$ & $-Q_{2}$ & 0 & 0 \\
\hline$Q_{3}$ & 0 & 0 & 0 \\
\hline
\end{tabular}

Then $L_{3}=\left\{Q_{1}, Q_{2}, Q_{3}\right\}$ is a solvable Lie algebra of (6).

Case 3 If $b$ are arbitrary constants not equal zero, and $a=c=d=0$ then the Equation (6) classified to Lie algebra of the form

$$
\begin{gathered}
Q_{1}=t \frac{\partial}{\partial t}, \quad Q_{2}=\frac{\partial}{\partial t}, \quad Q_{3}=t x\left(\frac{\partial}{\partial x}+\frac{1}{2} \frac{\partial}{\partial u}\right) \\
Q_{4}=x\left(\frac{\partial}{\partial x}+\frac{1}{2} \frac{\partial}{\partial u}\right), \quad Q_{5}=\frac{\partial}{\partial u}, \\
Q_{6}=-\frac{1}{b} g \frac{\partial}{\partial t}+[h-b x \xi] \frac{\partial}{\partial x}-\left[\frac{1}{b} h_{t}+\frac{b}{2} x \xi\right] \frac{\partial}{\partial u} .
\end{gathered}
$$

where $Q_{6}$ is an infinite dimensional Lie subalgebra. The commutator table is

\begin{tabular}{|c|l|c|l|c|l|}
\hline & $Q_{1}$ & $Q_{2}$ & $Q_{3}$ & $Q_{4}$ & $Q_{5}$ \\
\hline$Q_{1}$ & 0 & $-Q_{2}$ & $Q_{3}$ & 0 & 0 \\
\hline$Q_{2}$ & $Q_{2}$ & 0 & $Q_{4}$ & 0 & 0 \\
\hline$Q_{3}$ & $-Q_{3}$ & $-Q_{4}$ & 0 & 0 & 0 \\
\hline$Q_{4}$ & 0 & 0 & 0 & 0 & 0 \\
\hline$Q_{5}$ & 0 & 0 & 0 & 0 & 0 \\
\hline
\end{tabular}

Then $L_{4}=\left\{Q_{1}, Q_{2}, Q_{3}, Q_{4}, Q_{5}\right\}$ is a solvable Lie algebra of (6). 


\subsection{Second order neutral delay differential equations (NDDEs)}

Here we render a classification scheme for second order neutral delay differential equations of the form

$$
x^{\prime \prime}=f\left(t, x, x_{\tau}, x^{\prime}, x_{\tau}^{\prime}, x_{\tau}^{\prime \prime}\right)
$$

to solvable Lie algebra.

The following lemma is used to complete the classification.

Lemma 3.2 The second order neutral delay differential equations (10), containing the infinitesimal generator $\xi$ that obeys periodic property is given by,

$$
\xi(t, x)=\xi^{\tau}\left(t-\tau, x_{\tau}\right)
$$

\section{Proof}

First the canonical Lie-Bäcklund operator to the seven-dimensional space of variables $\left(t, x, x_{\tau}, x^{\prime}, x_{\tau}^{\prime}, x^{\prime \prime}, x_{\tau}^{\prime \prime}\right)$ is searched, which yields

$$
\tilde{X}_{\beta}=\zeta^{x} \frac{\partial}{\partial x}+\zeta^{x_{\tau}} \frac{\partial}{\partial x_{\tau}}+\zeta^{x^{\prime}} \frac{\partial}{\partial x^{\prime}}+\zeta^{x_{\tau}^{\prime}} \frac{\partial}{\partial x_{\tau}^{\prime}}+\zeta^{x^{\prime \prime}} \frac{\partial}{\partial x^{\prime \prime}}+\zeta^{x_{\tau}^{\prime \prime}} \frac{\partial}{\partial x_{\tau}^{\prime \prime}},
$$

where $\zeta=\eta-x^{\prime} \xi$, then

$$
\begin{aligned}
& \zeta^{x}\left(t, x, x^{\prime}\right)=\eta(t, x)-x^{\prime} \xi(t, x) . \\
& \zeta^{x_{\tau}}\left(t, x_{\tau}, x_{\tau}^{\prime}\right)=\eta^{\tau}\left(t-\tau, x_{\tau}\right)-x_{\tau}^{\prime} \xi^{\tau}\left(t-\tau, x_{\tau}\right) . \\
& \zeta^{x^{\prime}}\left(t, x, x^{\prime}, x^{\prime \prime}\right)=D\left(\zeta_{1}\right)=\eta_{t}(t, x)+\left[\eta_{x}(t, x)-\xi_{t}(t, x)\right] x^{\prime}-\xi_{x}(t, x)\left(x^{\prime}\right)^{2}-\xi(t, x) x^{\prime \prime} . \\
& \zeta^{x_{\tau}^{\prime}}\left(t, x_{\tau}, x_{\tau}^{\prime}, x_{\tau}^{\prime \prime}\right)=D\left(\zeta_{1}^{\tau}\right)=\eta_{t}^{\tau}\left(t-\tau, x_{\tau}\right)+\left[\eta_{x}^{\tau}\left(t-\tau, x_{\tau}\right)-\xi_{t}^{\tau}\left(t-\tau, x_{\tau}\right)\right] x_{\tau}^{\prime}-\xi_{x}^{\tau}\left(t-\tau, x_{\tau}\right)\left(x_{\tau}^{\prime}\right)^{2} \\
& -\xi^{\tau}\left(t-\tau, x_{\tau}\right) x_{\tau}^{\prime \prime} \\
& \zeta^{x^{\prime \prime}}\left(t, x, x^{\prime}, x^{\prime \prime}, x^{\prime \prime \prime}\right)=D\left(\zeta_{2}\right)=\eta_{t t}(t, x)+\left[2 \eta_{t x}(t, x)-\xi_{t t}(t, x)\right] x^{\prime}+\left[\eta_{x x}(t, x)-2 \xi_{t x}(t, x)\right]\left(x^{\prime}\right)^{2} \\
& -\xi_{x x}(t, x)\left(x^{\prime}\right)^{3}+\left[\eta_{x}(t, x)-2 \xi_{t}(t, x)\right] x^{\prime \prime}-3 \xi_{x}(t, x) x^{\prime} x^{\prime \prime}-\xi(t, x) x^{\prime \prime \prime} . \\
& \zeta^{x_{\tau}^{\prime \prime}}\left(t, x_{\tau}, x_{\tau}^{\prime}, x_{\tau}^{\prime \prime}, x_{\tau}^{\prime \prime \prime}\right)=D\left(\zeta_{2}^{\tau}\right)=\eta_{t t}^{\tau}\left(t-\tau, x_{\tau}\right)+\left[2 \eta_{t x}^{\tau}\left(t-\tau, x_{\tau}\right)-\xi_{t t}^{\tau}\left(t-\tau, x_{\tau}\right)\right] x_{\tau}^{\prime}+ \\
& {\left[\eta_{x x}^{\tau}\left(t-\tau, x_{\tau}\right)-2 \xi_{t x}^{\tau}\left(t-\tau, x_{\tau}\right)\right]\left(x_{\tau}^{\prime}\right)^{2}-\xi_{x x}^{\tau}\left(t-\tau, x_{\tau}\right)\left(x_{\tau}^{\prime}\right)^{3}+\left[\eta_{x}^{\tau}\left(t-\tau, x_{\tau}\right)-2 \xi_{t}^{\tau}\left(t-\tau, x_{\tau}\right)\right] x_{\tau}^{\prime \prime}-} \\
& 3 \xi_{x}^{\tau}\left(t-\tau, x_{\tau}\right) x_{\tau}^{\prime} x_{\tau}^{\prime \prime}-\xi^{\tau}\left(t-\tau, x_{\tau}\right) x_{\tau}^{\prime \prime \prime} .
\end{aligned}
$$

$D$ is the total derivative with respect to $t$. The determining equation of (10) is

$$
\tilde{X}_{\beta}=\left.\left(x^{\prime \prime}-f\left(t, x, x_{\tau}, x^{\prime}, x_{\tau}^{\prime}, x_{\tau}^{\prime \prime}\right)\right)\right|_{(10)}=0 .
$$


Note that (11) have to be satisfied for any solution of (10).

Let $x^{\prime \prime \prime}=f_{t}+x^{\prime} f_{x}+x_{\tau}^{\prime} f_{x_{\tau}}+x^{\prime \prime} f_{x^{\prime}}+x_{\tau}^{\prime \prime} f_{x_{\tau}^{\prime}}, x_{\tau}^{\prime \prime \prime}=f_{t}^{\tau}+x^{\prime} f_{x}^{\tau}+x_{\tau}^{\prime} f_{x_{\tau}}^{\tau}+x^{\prime \prime} f_{x^{\prime}}^{\tau}+$ $x_{\tau}^{\prime \prime} f_{x_{\tau}^{\prime}}^{\tau}, x^{\prime \prime}=f$ and $x_{\tau}^{\prime \prime}=f_{\tau}$, then (11) become

$$
\begin{aligned}
& \xi_{x x}^{\tau} f_{f_{\tau}}\left(x_{\tau}^{\prime}\right)^{3}-\xi_{x x}\left(x^{\prime}\right)^{3}+\left[\eta_{x x}-2 \xi_{t x}+\xi_{x} f_{x^{\prime}}\right]\left(x^{\prime}\right)^{2}+\left[\xi_{x}^{\tau} f_{x_{\tau}^{\prime}}+\left(2 \xi_{t x}^{\tau}-\eta_{x x}^{\tau}\right) f_{f_{\tau}}\right]\left(x_{\tau}^{\prime}\right)^{2}+ \\
& {\left[2 \eta_{t x}-\xi_{t t}+\left(\xi_{t}-\eta_{x}\right) f_{x^{\prime}}-3 \xi_{x} f+\xi^{\tau} f_{x}^{\tau} f_{f_{\tau}}\right] x^{\prime}+\left[\left(\xi_{t}^{\tau}-\eta_{x}^{\tau}\right) f_{x_{\tau}^{\prime}}+\left(\xi_{t t}^{\tau}-2 \eta_{t x}^{\tau}\right) f_{f_{\tau}}+3 \xi_{x}^{\tau} f f_{f_{\tau}}+\xi^{\tau} f_{x_{\tau}}^{\tau} f_{f_{\tau}}\right] x_{\tau}^{\prime}} \\
& +\eta_{t t}+\left(\eta_{x}-2 \xi_{t}\right) f-\xi f_{t}-\eta f_{x}-\eta^{\tau} f_{x_{\tau}}-\eta_{t} f_{x^{\prime}}-\eta_{t}^{\tau} f_{x_{\tau}^{\prime}}-\xi f f_{x}+\xi f f_{x^{\prime}}-\eta_{t t}^{\tau} f_{f_{\tau}}+\left(2 \xi_{t}^{\tau}-\eta_{x}^{\tau}\right) f_{\tau} f_{f_{\tau}}+ \\
& \quad \xi^{\tau} f_{t}^{\tau} f_{f_{\tau}}+\xi^{\tau} f f_{x^{\prime}}^{\tau} f_{f_{\tau}}+\xi^{\tau} f_{\tau} f_{x_{\tau}^{\prime}}^{\tau} f_{f_{\tau}}+\left(\xi^{\tau}-\xi\right) f_{x_{\tau}} x_{\tau}^{\prime}+\left(\xi^{\tau}-\xi\right) f_{\tau} f_{x_{\tau}^{\prime}}=0 .
\end{aligned}
$$

Where $f_{\tau}=f\left(t-\tau, x_{\tau}, x_{2 \tau}, x_{\tau}^{\prime}, x_{2 \tau}^{\prime}\right), x_{2 \tau}=x(t-2 \tau)$ and $x_{2 \tau}^{\prime}=x^{\prime}(t-2 \tau)$. By virtue of the Cauchy problem (see [23]), one can account the variables $t, x, x_{\tau}, x^{\prime}, x_{\tau}^{\prime}, x_{2 \tau}$ and $x_{2 \tau}^{\prime}$ in (12) as arbitrary variables.

For the case $f_{x_{\tau}^{\prime}} \neq 0$, we can split the determining equation (12) with respect to $x_{2 \tau}^{\prime}$. This implies that $\xi=\xi^{\tau}$.

If $f_{x_{\tau}^{\prime}}=0$, then the assumption of DDE implies $f$ must depend on the delay terms, i.e. $f_{x_{\tau}} \neq 0$. Splitting (12) with respect to $x_{\tau}^{\prime}$, we also get $\xi=\xi^{\tau}$. This shows the periodic property of $\xi$.

Lemma (3.2) allows one to rewrite (11) in the form

$$
\left.\tilde{X}\left(x^{\prime \prime}-f\left(t, x, x_{\tau}, x^{\prime}, x_{\tau}^{\prime}, x_{\tau}^{\prime \prime}\right)\right)\right|_{(10)}=0
$$

Where

$$
\tilde{X}=\tilde{X}_{\beta}+\xi D=\xi \frac{\partial}{\partial t}+\eta^{x} \frac{\partial}{\partial x}+\eta^{x_{\tau}} \frac{\partial}{\partial x_{\tau}}+\eta^{x^{\prime}} \frac{\partial}{\partial x^{\prime}}+\eta^{x_{\tau}^{\prime}} \frac{\partial}{\partial x_{\tau}^{\prime}}+\eta^{x^{\prime \prime}} \frac{\partial}{\partial x^{\prime \prime}}+\eta^{x_{\tau}^{\prime \prime}} \frac{\partial}{\partial x_{\tau}^{\prime \prime}},
$$

and

$$
\begin{gathered}
\eta^{x_{\tau}^{\prime \prime}}\left(t, x_{\tau}, x_{\tau}^{\prime}, x_{\tau}^{\prime \prime}\right)=\eta_{2}^{\tau}\left(t, x_{\tau}, x_{\tau}^{\prime}, x_{\tau}^{\prime \prime}\right)=\eta_{t t}^{\tau}\left(t-\tau, x_{\tau}\right)+\left[2 \eta_{t x}^{\tau}\left(t-\tau, x_{\tau}\right)-\xi_{t t}^{\tau}\left(t-\tau, x_{\tau}\right)\right] x_{\tau}^{\prime} \\
+\left[\eta_{x x}^{\tau}\left(t-\tau, x_{\tau}\right)-2 \xi_{t x}^{\tau}\left(t-\tau, x_{\tau}\right)\right]\left(x_{\tau}^{\prime}\right)^{2}-\xi_{x x}^{\tau}\left(t-\tau, x_{\tau}\right)\left(x_{\tau}^{\prime}\right)^{3}+\left[\eta_{x}^{\tau}\left(t-\tau, x_{\tau}\right)-\right. \\
\left.2 \xi_{t}^{\tau}\left(t-\tau, x_{\tau}\right)\right] x_{\tau}^{\prime \prime}-3 \xi_{x}^{\tau}\left(t-\tau, x_{\tau}\right) x_{\tau}^{\prime} x_{\tau}^{\prime \prime}
\end{gathered}
$$

The difference between the generator $\tilde{X}$ and $\tilde{X}_{\beta}$ is, the generator $\tilde{X}$ acts in the space of variables $\left(t, x, x_{\tau}, x^{\prime}, x_{\tau}^{\prime}, x^{\prime \prime}, x_{\tau}^{\prime \prime}\right)$, whereas the latter coefficients of the operator include the derivatives $x_{\tau}^{\prime \prime \prime}$ and $x^{\prime \prime \prime}$.

Now, using the invariant manifold theorem, one obtains the generator $\tilde{X}$ [?]. Consequently, the equation (11) readily implies that the manifold defined by $(10)$ is an invariant manifold of $\tilde{X}$. 
The general form of second order neutral delay differential equation is given by,

$$
x^{\prime \prime}(t)+a(t) x^{\prime \prime}(t-\tau)+b(t) x^{\prime}(t)+c(t) x^{\prime}(t-\tau)+d(t) x(t)+e(t) x(t-\tau)=0,
$$

where $a, b, d, e$ are arbitrary coefficients.

The general infinitesimal generator associated with (15) can be expressed as,

$$
X=\xi(t, x) \frac{\partial}{\partial t}+\eta(t, x) \frac{\partial}{\partial x}+\eta^{\tau}\left(t-\tau, x_{\tau}\right) \frac{\partial}{\partial x_{\tau}} .
$$

Using Equation (13) the second order extension yields,

$$
\begin{gathered}
X^{(2)}=X+\eta_{1}\left(t, x, x^{\prime}\right) \frac{\partial}{\partial x^{\prime}}+\eta_{2}\left(t, x, x^{\prime}, x^{\prime \prime}\right) \frac{\partial}{\partial x^{\prime \prime}}+\eta_{1}^{\tau}\left(t-\tau, x_{\tau}, x_{\tau}^{\prime}\right) \frac{\partial}{\partial x_{\tau}^{\prime}} \\
+\eta_{2}^{\tau}\left(t-\tau, x_{\tau}, x_{\tau}^{\prime}, x_{\tau}^{\prime \prime}\right) \frac{\partial}{\partial x_{\tau}^{\prime \prime}} .
\end{gathered}
$$

Substituting (17) to (15), one recovers the invariance conditions as,

$$
\eta_{2}+a \eta_{2}^{\tau}+b \eta_{1}+c \eta_{1}^{\tau}+d \eta+e \eta^{\tau}=0
$$

Substituting (14), we get

$$
\begin{gathered}
\eta_{t t}+\left[2 \eta_{t x}-\xi_{t t}\right] x^{\prime}+\left[\eta_{x x}-2 \xi_{t x}\right]\left(x^{\prime}\right)^{2}-\xi_{x x}\left(x^{\prime}\right)^{3}+\left[\eta_{x}-2 \xi_{t}\right] x^{\prime \prime}-3 \xi_{x} x^{\prime} x^{\prime \prime}+a\left[\eta_{t t}^{\tau}+\left[2 \eta_{t x}^{\tau}-\xi_{t t}^{\tau}\right] x_{\tau}^{\prime}\right. \\
\left.+\left[\eta_{x x}^{\tau}-2 \xi_{t x}^{\tau}\right]\left(x_{\tau}^{\prime}\right)^{2}-\xi_{x x}^{\tau}\left(x_{\tau}^{\prime}\right)^{3}+\left[\eta_{x}^{\tau}-2 \xi_{t}^{\tau}\right] x_{\tau}^{\prime \prime}-3 \xi_{x}^{\tau} x_{\tau}^{\prime} x_{\tau}^{\prime \prime}\right]+b\left[\eta_{t}+\left[\eta_{x}-\xi_{t}\right] x^{\prime}-\xi_{x}\left(x^{\prime}\right)^{2}\right]+c\left[\eta_{t}^{\tau}+\right. \\
\left.\left[\eta_{x}^{\tau}-\xi_{t}^{\tau}\right] x_{\tau}^{\prime}-\xi_{x}^{\tau}\left(x_{\tau}^{\prime}\right)^{2}\right]+d \eta+e \eta^{\tau}=0 .
\end{gathered}
$$

Equating the first and second order coefficients of the various monomials of $x$ and $x_{\tau}$, the following determining equations for the symmetry group of (15) are obtained.

Table 2: The determining equations for the symmetry group of (15).

\begin{tabular}{|c|l|c|}
\hline monomial & coefficient & number of equation \\
\hline 1 & $\eta_{t t}+a \eta_{t t}^{\tau}+b \eta_{t}+c \eta_{t}^{\tau}+d \eta+e \eta^{\tau}=0$ & $a_{1}$ \\
\hline$x^{\prime}$ & $2 \eta_{t x}-\xi_{t t}+b\left[\eta_{x}-\xi_{t}\right]=0$ & $a_{2}$ \\
\hline$\left(x^{\prime}\right)^{2}$ & $\eta_{x x}-2 \xi_{t x}-b \xi_{x}=0$ & $a_{3}$ \\
\hline$\left(x^{\prime}\right)^{3}$ & $\xi_{x x}=0$ & $a_{4}$ \\
\hline$x^{\prime} x^{\prime \prime}$ & $\xi_{x}=0$ & $a_{5}$ \\
\hline
\end{tabular}




\begin{tabular}{|c|l|c|}
\hline monomial & coefficient & number of equation \\
\hline$x^{\prime \prime}$ & $\eta_{x}-2 \xi_{t}=0$ & $a_{6}$ \\
\hline$x_{\tau}^{\prime}$ & $a\left[2 \eta_{t x}^{\tau}-\xi_{t t}^{\tau}\right]+c\left[\eta_{x}^{\tau}-\xi_{t}^{\tau}\right]=0$ & $a_{7}$ \\
\hline$\left(x_{\tau}^{\prime}\right)^{2}$ & $a\left[\eta_{x x}^{\tau}-2 \xi_{t x}^{\tau}\right]-c \xi_{x}^{\tau}=0$ & $a_{8}$ \\
\hline$\left(x^{\prime}\right)^{3}$ & $a \xi_{x x}^{\tau}=0$ & $a_{9}$ \\
\hline$x_{\tau}^{\prime \prime}$ & $a\left[\eta_{x}^{\tau}-2 \xi_{t}^{\tau}\right]=0$ & $a_{10}$ \\
\hline$x_{\tau}^{\prime} x_{\tau}^{\prime \prime}$ & $a \xi_{x}^{\tau}=0$ & $a_{11}$ \\
\hline
\end{tabular}

In $\left(a_{5}\right), \xi$ does not depend on $x$. In $\left(a_{3}\right), \eta$ is linearly vary with $x$, following $\eta=g(t) x+h(t)$, where $g(t)$ and $h(t)$ are arbitrary functions. In $\left(a_{6}\right), \xi_{t}=\frac{1}{2} g$.

In $\left(a_{11}\right), \xi^{\tau}$ is independent of $x$, In lemma $(3.2), \xi=\xi^{\tau}$ then $\xi_{t}=\xi_{t}^{\tau}$, which implies that $\xi_{t}^{\tau}=\frac{1}{2} g(t)$. In $\left(a_{10}\right), \eta_{x}^{\tau}=2 \xi_{t}^{\tau}$, and $\eta_{x}^{\tau}=g(t)$, thus $\eta^{\tau}=g(t) x+$ $k(t-\tau)$, with $k(t-\tau)$ is arbitrary function.

Case 1 If $a, b, c, d$ and $e$ are arbitrary constants not equal to zero. Then using $\left(a_{1}\right)$ one gets $g_{t t} x+h_{t t}+a g_{t t} x+a k_{t t}+b g_{t} x+b h_{t}+c g_{t} x+c k_{t}+d g x+d h+$ $e g x+e k=0$. Equating the coefficients of the various monomials one obtains,

$$
\begin{aligned}
& (1+a) g_{t t}+(b+c) g_{t}+(d+e) g=0 . \\
& h_{t t}+a k_{t t}+b h_{t}+c k_{t}+d h+e k=0 .
\end{aligned}
$$

This implies that $g(t), h(t)$ and $k(t-\tau)$ are the solutions of (15). From (18), and since $g=2 \xi_{t}$, then

$$
\xi_{t}=-\frac{1+a}{d+e} \xi_{t t t}-\frac{b+c}{d+e} \xi_{t t}
$$

then

$$
\xi=-\frac{1+a}{d+e} \xi_{t t}-\frac{b+c}{d+e} \xi_{t}+c_{1} .
$$

Let $1+a=A, b+c=C$ and $d+e=E$, then,

$$
\xi=c_{1}-\frac{A}{2 E} g_{t}-\frac{C}{2 E} \text {. }
$$


Now,

$$
g=c_{2}-\frac{A}{C} g_{t}-\frac{2 E}{C} \xi
$$

From reduce to $\eta=\left(c_{2}-\frac{A}{C} g_{t}-\frac{2 E}{C} \xi\right) x+h$ and $\eta^{\tau}=\left(c_{2}-\frac{A}{C} g_{t}-\frac{2 E}{C} \xi\right) x+k$, where $c_{i}, i=1,2$ are arbitrary constants.

Recalling the general infinitesimal generator for (15) we get

$$
X=\xi \frac{\partial}{\partial t}+\eta \frac{\partial}{\partial x}+\eta^{\tau} \frac{\partial}{\partial x_{\tau}} .
$$

Let $x_{\tau}=u$, then

$$
X=\left[c_{1}-\frac{A}{2 E} g_{t}-\frac{C}{2 E}\right] \frac{\partial}{\partial t}+\left[\left(c_{2}-\frac{A}{C} g_{t}-\frac{2 E}{C} \xi\right) x+h\right] \frac{\partial}{\partial x}+\left[\left(c_{2}-\frac{A}{C} g_{t}-\frac{2 E}{C} \xi\right) x+k\right] \frac{\partial}{\partial u} .
$$

The Lie algebra of (15) is spanned by the following three infinitesimal generater corresponding to each parameters and is given by $c_{i}, i=1,2$

$$
\begin{gathered}
Q_{1}=\frac{\partial}{\partial t}, \quad Q_{2}=x\left(\frac{\partial}{\partial x}+\frac{\partial}{\partial u}\right), \\
Q_{3}=-\left(\frac{A}{2 E} g_{t}+\frac{C}{2 E} g\right) \frac{\partial}{\partial t}+\left[h(t)-x\left(\frac{A}{C} g_{t}+\frac{2 E}{C} \xi\right)\right] \frac{\partial}{\partial x}+\left[k(t)-x\left(\frac{A}{C} g_{t}+\frac{2 E}{C} \xi\right)\right] \frac{\partial}{\partial u},
\end{gathered}
$$

Where $Q_{3}$ is an infinite dimensional Lie subalgebra, $L_{2}=\left\{Q_{1}, Q_{2}\right\}$ is a solvable Lie algebra of (15).

Remark 3.3 The same result is obtained for d, or e equal to zero.

Case 2 For $a, b, c$ are arbitrary constants and $d=e=0$, the Lie algebra of (15) is spanned by

$$
Q_{1}=t x\left(\frac{\partial}{\partial x}+\frac{\partial}{\partial u}\right), \quad Q_{2}=x\left(\frac{\partial}{\partial x}+\frac{\partial}{\partial u}\right), \quad Q_{3}=t \frac{\partial}{\partial t}, \quad Q_{4}=\frac{\partial}{\partial t}, \quad Q_{5}=\frac{\partial}{\partial u}
$$

with an infinite dimensional Lie subalgebra gives,

$$
Q_{6}=\left(\frac{-A}{2 C} g\right) \frac{\partial}{\partial t}+\left[h-\frac{2 C}{A} \xi x\right] \frac{\partial}{\partial x}-\left[\frac{1}{c} h_{t}+\frac{a}{c} k_{t}+\frac{b}{c} h+\frac{2 C}{A} \xi x\right] \frac{\partial}{\partial u} .
$$

The commutator table can be written as, 


\begin{tabular}{|c|l|c|l|c|l|}
\hline & $Q_{1}$ & $Q_{2}$ & $Q_{3}$ & $Q_{4}$ & $Q_{5}$ \\
\hline$Q_{1}$ & 0 & 0 & $-Q_{1}$ & $-Q_{2}$ & 0 \\
\hline$Q_{2}$ & 0 & 0 & 0 & 0 & 0 \\
\hline$Q_{3}$ & $Q_{1}$ & 0 & 0 & $-Q_{4}$ & 0 \\
\hline$Q_{4}$ & $Q_{2}$ & 0 & $Q_{4}$ & 0 & 0 \\
\hline$Q_{5}$ & 0 & 0 & 0 & 0 & 0 \\
\hline
\end{tabular}

Now $L_{4}=\left\{Q_{1}, Q_{2}, Q_{3}, Q_{4}, Q_{5}\right\}$ is a solvable Lie algebra of Equation (15).

Remark 3.4 If $a, c$ are arbitrary constant not equal to one, and $b=d=$ $e=0$, we get the same result of Case 2.

The infinite dimensional Lie subalgebra is casted as,

$$
Q=-\frac{-A}{2 c} g \frac{\partial}{\partial t}+\left(h-\frac{2 c}{A} \xi x\right) \frac{\partial}{\partial x}-\left(\frac{1}{c} h_{t}+\frac{a}{c} k_{t}+x \xi\right) \frac{\partial}{\partial u} .
$$

Case 3 If $a=b=c=d=1$, and $e=0$ or $a=b=c=e=1$, and $d=0$, the solvable Lie algebra of (15) is spanned by

$$
\begin{gathered}
Q_{1}=\frac{\partial}{\partial t}+x\left(\frac{\partial}{\partial x}+\frac{\partial}{\partial u}\right) \\
Q_{2}=-\left(g_{t}+g\right) \frac{\partial}{\partial t}+\left[h-\left(g_{t}+\xi\right) x\right] \frac{\partial}{\partial x}+\left[k-\left(g_{t}+\xi\right) x\right] \frac{\partial}{\partial u} .
\end{gathered}
$$

Where $Q_{2}$ is an infinite dimensional Lie subalgebra.

Case 4 If $a=c=1$, and $b=d=e=0$, the Lie algebra of (15) is spanned by

$$
\begin{gathered}
Q_{1}=t \frac{\partial}{\partial t}+x t\left(\frac{\partial}{\partial x}+\frac{\partial}{\partial u}\right), \quad Q_{2}=\frac{\partial}{\partial t}+x\left(\frac{\partial}{\partial x}+\frac{\partial}{\partial u}\right), \quad Q_{3}=\frac{\partial}{\partial u}, \\
Q_{4}=-g \frac{\partial}{\partial t}+(h-\xi x) \frac{\partial}{\partial x}-\left(h_{t}+k_{t}+\xi x\right) \frac{\partial}{\partial u} .
\end{gathered}
$$

Where $Q_{4}$ is an infinite dimensional Lie subalgebra. The commutator table is represented as,

\begin{tabular}{|c|l|c|l|}
\hline & $Q_{1}$ & $Q_{2}$ & $Q_{3}$ \\
\hline$Q_{1}$ & 0 & $-Q_{2}$ & 0 \\
\hline$Q_{2}$ & $Q_{2}$ & 0 & 0 \\
\hline$Q_{3}$ & 0 & 0 & 0 \\
\hline
\end{tabular}


Then $L_{2}=\left\{Q_{1}, Q_{2}, Q_{3}\right\}$ is a solvable Lie algebra of (15).

Based on the above results we give the following algorithm

\subsection{Algorithm}

The procedure of classifying second order linear delay differential equations with constant coefficients to solvable Lie algebra is summarized as follows:

1. Write the delay differential equation in the solved form.

2. Select the general infinitesimal generator of DDEs.

3. Extend the infinitesimal generator acting on second order.

4. Apply the extended infinitesimal generator to the given delay differential equation to obtain invariance condition.

5. Substitute Equations (5) or (14) in the invariance condition conform to an infinitesimal symmetry.

6. Split up invariance conditions of the coefficients of the various monomials in the first and second orders of $x$ and $x_{\tau}$ for determining equations of the infinitesimal symmetry group.

7. Solve the determining equations for the infinitesimals $\xi, \eta$, and $\eta^{\tau}$.

8. Substitute the infinitesimals $\xi, \eta$, and $\eta^{\tau}$ in the generator.

9. Thus the Lie algebra of the given equation is spanned by the three infinitesimals generators corresponding to each parameter $c_{i}, i=1,2, \ldots, n$ with $c_{i}$ as arbitrary constant.

10. Compute the commutator table on the basis of Lie algebra.

11. Satisfy the inclusion property to achieve solvable Lie algebra. 
Table 3: Classification of second order linear delay differential equations with constant coefficients to solvable Lie algebra.

\begin{tabular}{|c|c|c|}
\hline $\begin{array}{l}\text { Second order LDDEs with } \\
\text { constant coefficients }\end{array}$ & Basis of Lie algebra & Solvable Lie algebra \\
\hline $\begin{array}{l}x^{\prime \prime}+a x^{\prime}+b x_{\tau}^{\prime}+c x+d x_{\tau}=0 \\
\text { or if } c=0 \text { or } d=0\end{array}$ & $\begin{array}{l}Q_{1}=\frac{\partial}{\partial t}, Q_{2}=x \frac{\partial}{\partial x}, Q_{3}= \\
x \frac{\partial}{\partial u}, \\
\text { with Lie subalgebra } Q_{4}= \\
-\left(\frac{1}{2 D} g_{t}+\frac{B}{2 D}\right) \frac{\partial}{\partial t}+(h(t)- \\
x\left(\frac{1}{B} g_{t}+\frac{D}{B} \xi\right) \frac{\partial}{\partial x}+(k- \\
x\left(\frac{1}{2 B} g_{t}+\frac{D}{2 B} \xi\right) \frac{\partial}{\partial u}\end{array}$ & $L_{3}^{1}$ \\
\hline$x^{\prime \prime}+x_{\tau}^{\prime}=0$ & $\begin{array}{l}Q_{1}=t \frac{\partial}{\partial t}+x t\left(\frac{\partial}{\partial x} \frac{1}{2} \frac{\partial}{\partial u}\right), Q_{2}= \\
\frac{\partial}{\partial t}+x\left(\frac{\partial}{\partial x}+\frac{1}{2} \frac{\partial}{\partial u}\right), Q_{3}=\frac{\partial}{\partial u}, \\
\text { with Lie subalgebra } Q_{4}= \\
-g \frac{\partial}{\partial t}+(h-x \xi) \frac{\partial}{\partial x}-\left(h_{t}+\right. \\
\left.\frac{1}{2} x \xi\right) \frac{\partial}{\partial u}\end{array}$ & $L_{3}^{2}$ \\
\hline $\begin{array}{l}x^{\prime \prime}+a x_{\tau}^{\prime \prime}+b x^{\prime}+c x_{\tau}^{\prime}=0 \\
\text { Or } x^{\prime \prime}+a x_{\tau}^{\prime \prime}+c x_{\tau}^{\prime}=0\end{array}$ & $\begin{array}{l}Q_{1}=t x\left(\frac{\partial}{\partial x}+\frac{\partial}{\partial u}\right), Q_{2}= \\
x\left(\frac{\partial}{\partial x}+\frac{\partial}{\partial u}\right), Q_{3}=t \frac{\partial}{\partial t}, Q_{4}= \\
\frac{\partial}{\partial t}, Q_{5}=\frac{\partial}{\partial u}, \\
\text { with Lie subalgebra } \\
Q_{6}=-\frac{A}{2 C} g \frac{\partial}{\partial t}+[h- \\
\left.\frac{2 C}{A} x \xi\right] \frac{\partial}{\partial x}-\left[\frac{1}{c} h_{t}+\frac{a}{c} k_{t}+\frac{b}{c} h+\right. \\
\left.\frac{2 C}{A} x \xi\right] \frac{\partial}{\partial u}\end{array}$ & $L_{5}^{3}$ \\
\hline
\end{tabular}




\begin{tabular}{|c|c|c|}
\hline $\begin{array}{c}\text { Second order LDDEs with } \\
\text { constant coefficients }\end{array}$ & Basis of Lie algebra & $\begin{array}{c}\text { Solvable } \\
\text { Lie algebra }\end{array}$ \\
\hline$x^{\prime \prime}+b x_{\tau}^{\prime}=0$ & $\begin{array}{l}Q_{1}=t \frac{\partial}{\partial t}, Q_{2}=\frac{\partial}{\partial t}, Q_{3}=t x\left(\frac{\partial}{\partial x}+\right. \\
\left.\frac{1}{2} \frac{\partial}{\partial u}\right), Q_{4}=x\left(\frac{\partial}{\partial x}+\frac{1}{2} \frac{\partial}{\partial u}\right), Q_{5}= \\
\frac{\partial}{\partial u}, \text { with Lie subalgebra } Q_{6}= \\
-\frac{1}{b} g \frac{\partial}{\partial t}+[h-b x \xi] \frac{\partial}{\partial x}-\left[\frac{1}{b} h_{t}-\frac{b}{2} x \xi\right] \frac{\partial}{\partial u}\end{array}$ & $L_{5}^{4}$ \\
\hline $\begin{array}{l}x^{\prime \prime}+a x_{\tau}^{\prime \prime}+b x^{\prime}+c x_{\tau}^{\prime}+d x+ \\
e x_{\tau}=0 \text { or if } d=0 \text { or } e=0\end{array}$ & $\begin{array}{l}Q_{1}=\frac{\partial}{\partial t}, Q_{2}=x\left(\frac{\partial}{\partial x}+\frac{\partial}{\partial u}\right) \\
\text { with Lie subalgebra } Q_{3}= \\
-\left(\frac{A}{2 E} g_{t}+\frac{C}{2 E} g\right) \frac{\partial}{\partial t}+\left[h-\left(\frac{A}{C} g_{t}+\right.\right. \\
\left.\left.\frac{2 E}{C} \xi\right) x\right] \frac{\partial}{\partial x}+\left[k-\left(\frac{A}{C} g_{t}+\frac{2 E}{C} \xi\right) x\right] \frac{\partial}{\partial u}\end{array}$ & $L_{2}^{5}$ \\
\hline$x^{\prime \prime}+x_{\tau}^{\prime \prime}+x_{\tau}^{\prime}=0$ & $\begin{array}{l}Q_{1}=t \frac{\partial}{\partial t}+x t\left(\frac{\partial}{\partial x}+\frac{\partial}{\partial u}\right), Q_{2}= \\
\frac{\partial}{\partial t}+x\left(\frac{\partial}{\partial x}+\frac{\partial}{\partial u}\right), Q_{3}=\frac{\partial}{\partial u}, \text { with } \\
\text { Lie subalgebra } Q_{4}=-g \frac{\partial}{\partial t}+(h- \\
\xi x) \frac{\partial}{\partial x}-\left(h_{t}+k_{t}+\xi x\right) \frac{\partial}{\partial u}\end{array}$ & $L_{2}^{6}$ \\
\hline$x^{\prime \prime}+x_{\tau}^{\prime \prime}+x^{\prime}+x_{\tau}^{\prime}+x=0$ & $\begin{array}{l}Q_{1}=\frac{\partial}{\partial t}+x\left(\frac{\partial}{\partial x}+\frac{\partial}{\partial u}\right), \text { with Lie sub- } \\
\text { algebra }\end{array}$ & $L_{1}^{7}$ \\
\hline$x^{\prime \prime}+x_{\tau}^{\prime \prime}+x^{\prime}+x_{\tau}^{\prime}+x_{\tau}=0$ & $\begin{array}{l}Q_{2}=-\left(g_{t}+g\right) \frac{\partial}{\partial t}+\left[h-\left(g_{t}+\right.\right. \\
\xi) x] \frac{\partial}{\partial x}+\left[k-\left(g_{t}+\xi\right) x\right] \frac{\partial}{\partial u}\end{array}$ & \\
\hline
\end{tabular}

Here $g=g(t), h=h(t)$, and $k=k(t-\tau)$ are arbitrary solutions of the given equation, $A=1+a, B=a+\frac{b}{2}, C=b+c, D=c+\frac{d}{2}, E=d+e$. And $x=x(t), x_{\tau}=x(t-\tau), x^{\prime}=x^{\prime}(t), x_{\tau}^{\prime}=x^{\prime}(t-\tau), x^{\prime \prime}=x^{\prime \prime}(t)$ and $x_{\tau}^{\prime \prime}=x^{\prime \prime}(t-\tau)$. In $L_{m}^{n}, m$ is dimension of solvable Lie algebra and $n$ is the 
number in the table.

\section{Conclusion}

We developed a new approach for classifying second order linear DDEs with constant coefficients of the form

$$
x^{\prime \prime}=f\left(t, x, x_{\tau}, x^{\prime}, x_{\tau}^{\prime}\right),
$$

to solvable Lie algebra. The method is further productively applied to second order neutral DDEs of the form

$$
x^{\prime \prime}=f\left(t, x, x_{\tau}, x^{\prime}, x_{\tau}^{\prime}, x_{\tau}^{\prime \prime}\right) .
$$

Interestingly, the change in the space variables are not considered because the DDEs do not acquire an equivalent transformation related to this change. Furthermore, the general infinitesimal generator of DDEs is used to determine the equations for the same symmetry group. The equations are successfully solved to span the solvable Lie algebras by satisfying the inclusion property. This unique classification scheme is validated using the emerging neutral DDEs which is due to the failure of retarded DDEs in explaining problems related to natural phenomena such as motion of radiating electrons, population growth, the spread of epidemics and in networks containing lossless transmission lines. The flexibility of the proposed method suggests that it may further be generalized to classify second order linear DDE with functional coefficients, as well as non-linear DDEs.

Acknowledgements. The authors are thankful to Research Management Center (UTM) and Ministry of Higher Education (MOHE), Malaysia for financial support through research grants of votes 4F127 and 07J77. Laheeb is grateful to Ministry of Higher Education, Iraq for providing study leave and fellowship.

\section{References}

[1] P. Pue-on, Group classification of second-order delay differential equations, Commun Nonlinear Sci Numer Simul, 15 (2009), 1444 - 1453.

[2] S.A. Campbell, Introduction to delay differential equations, Ph.D. Thesis: University of Waterloo, Canada, 2007.

[3] R.D. Driver, Ordinary and delay differential equations, Springer New, York, 1977. 
[4] J.E. Forde, Delay differential equation models in mathematical biology, Ph.D. Thesis: University of Michigan, USA, 2005.

[5] K. Schmitt, Delay and functional differential equations and their applications, New York and London, 1972.

[6] H.L. Smith, An introduction to delay differential equations with applications to the life sciences, Texts in Applied Mathematics, Springer, New York, 2011.

[7] J. Yang, S. Liang and Y. Zhang, Travelling waves of a delayed SIR Epidemic model with nonlinear incidence rate and spatial diffusion, Plos one journal, 6 (2011),e21128.

[8] V. Kolmanovskii and A. Myshkis, Applied theory of functional differential equations, Kluwer Academic Publishers, Netherlands, 1992.

[9] J. Cushing, Integrodifferential equations and delay models in popluation dynamics, volume 20 of Lecture notes in biomathematics, Springer-Verlag, Berlin; New York, 1977.

[10] A. Bellen, M. Zennaro, Numerical methods for delay differential equations. Numerical mathematics and scientific computation, Clarendon Press, Oxford University Press, New York, 2003.

[11] J.J. Batzel and H.T. Tran, Stability of the human respiratory control system I, Analysis of a two-dimensional delay state-space model, J Math Biol, 41 (2000), 45-79.

[12] T.K. Nagy, G. Stepan and F.C. Moon, Subcritical Hopf bifurcation in the delay equation model for machine tool vibrations, Nonlinear Dyn, 26 (2011), 121-42.

[13] F. Oliveri, Lie symmetries of differential equations: classical results and recent contributions, Symmetry Journal, 2 (2010), 658-706.

[14] G.W. Bluman GW and S. Kumei, Symmetries and differential equations, Sprinder, New York, 1989.

[15] J.M. Hill, Solution of differential equation by means of one-parameter groups, London, 1982.

[16] P. J. Olver, Application of Lie groups to differential equations, (2nd ed), Springer, Verlag, New York, 1993.

[17] N. H. Ibragimov, Elementary Lie group analysis and ordinary differential equations, Wiley, London, 1999. 
[18] J. Thanthanuch and S.V. Meleshko, On definition of an admitted Lie group for functional differential equations, Commun Nonlinear Sci Numer Simul, 9 (2004), 117-25.

[19] V.M. Boyko, R.O. Popovych and N.M. Shapoval, Lie symmetries of systems of second-order linear ordinary differential equations with constant coefficients, Journal of Mathematical Analysis and Applications, 397 (2012), 434-440.

[20] S. Moyo, S.V. Meleshko and G.F. Oguis, Complete group classification of systems of two linear second-order ordinary differential equations, Commun Nonlinear Sci Numer Simul, 18 (2013), 2972-2983.

[21] $\tilde{C}$. Andreas, Lie algebras and representation theory, Universität Wien, Nordbergstr, 2009.

[22] M. Humi and W. Miller, Second course in ordinary differential equations for scientists and engineers, Springer, New York, 1988.

[23] R.J. Thelwell and P.G. Warne, Cauchy-kowaLlevski and polynomial ordinary differential equations, Department of Mathematics and Statistics, James Madison University, Harrisonburg, USA, 2011.

\section{Received: April 7, 2014}

